\title{
Staphylococcus aureus-induced septic arthritis of the ankle related to malum perforans in a diabetes patient
}

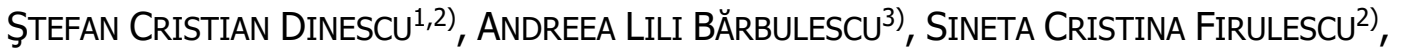 \\ ANDREEA BEATRICE CHISĂLĂU4), CRISTINA DORINA PÂRVĂNESCU4), PAULINA LUCIA CiUREA ${ }^{1,2)}$, \\ RALUCA ElENA SANDU ${ }^{5}$, AdINA TURCU-ŞTIOLICĂ6), MiHAIL VIRGIL BOLDEANU ${ }^{7}$ ), \\ ELENA MĂDĂLINA VinTILĂ ${ }^{8}$, FLORIN LIVIU GHERGHINA ${ }^{9)}$, ANANU FLORENTIN VREJU ${ }^{1,2)}$
}

1) Department of Rheumatology, University of Medicine and Pharmacy of Craiova, Romania
2)Department of Rheumatology, Emergency County Hospital, Craiova, Romania
3) Department of Pharmacology, University of Medicine and Pharmacy of Craiova, Romania
4)PhD Student, University of Medicine and Pharmacy of Craiova, Romania
5) Department of Biochemistry, University of Medicine and Pharmacy of Craiova, Romania
6) Department of Biostatistics, University of Medicine and Pharmacy of Craiova, Romania
7)Department of Immunology, University of Medicine and Pharmacy of Craiova, Romania
8) Department of Gastroenterology, Emergency County Hospital, Craiova, Romania
9) Department of Physiotherapy, University of Medicine and Pharmacy of Craiova, Romania

\begin{abstract}
Septic arthritis (SA) is a less common joint pathology with potentially fatal outcome. It is considered a medical emergency, in which prompt diagnosis and differentiation of bacterial etiology is essential for appropriate management. The knee is the most prevalent site for SA ( $\sim 50 \%$ of cases), followed by hip, shoulder, and elbow. Early intervention requires an accurate diagnosis and imaging techniques enable both a positive diagnosis, as well as arthrocentesis and liquid analysis, the "gold standard" criteria. We report the case of a 70-year-old patient, with history of rheumatoid arthritis (RA), diabetes mellitus (DM) and persistent left malum perforans in the last year, with development of a severe and debilitating Staphylococcus aureus-related SA of the left ankle, which posed significant therapeutic challenges. He developed a plantar lesion at the ball of the left foot, in the past one year, which was labeled as malum perforans in the setting of DM. Musculoskeletal ultrasound was the primary imaging technique used to define the location and extent of the infectious process. Cultures drawn from the tissue were positive for $S$. aureus. After an antibiotic course, the apparent infectious features were remitted but the long-lasting open wound failed to improve. Antibiotic therapy was initiated in accordance with culture sensibility tests but short- and long-term outcome was unfavorable with both treatment unresponsiveness and comorbidity burden posing considerable difficulties. The association and interrelation between different comorbidities (such as hypertension, diabetes, or obesity), chronic systemic inflammation (e.g., C-reactive protein level, disease activity), and RA medication is sometimes difficult to understand and to address in daily practice, and this case report highlights multiple toils encountered in a SA patient with RA on immunosuppressive therapy and complicated DM.
\end{abstract}

Keywords: septic arthritis, imaging methods, rheumatoid arthritis, diabetes.

\section{Introduction}

Septic arthritis (SA) is a less common joint pathology with potentially fatal outcome. It is considered a medical emergency, in which prompt diagnosis and differentiation of bacterial etiology is essential for appropriate management. The knee is the most prevalent site for SA ( $\sim 50 \%$ of cases), followed by hip, shoulder, and elbow [1]. SA develops either through hematogenous spread, direct inoculation, or extension from a contiguous infected tissue. Based on etiology, SA is generally divided in gonococcal and nongonococcal arthritis. Of the latter, most cases involve a Staphylococcus aureus (S. aureus) or Streptococcus species (Streptococcus spp.) infection. Multiple factors related to both host immunity and bacterial pathogenesis influence the severity of the disease [2]. Typical risk factors for developing SA include age $>80$ years, diabetes mellitus (DM), rheumatoid arthritis (RA), joint surgery $(<3$ months ago), hip or knee prosthesis, skin or human immunodeficiency virus (HIV) infection [1]. In contrast to gonococcal arthritis, non-gonococcal forms have a lower response rate to antibiotics and poorer prognosis. Overall, mortality rate in treated in-hospital SA patients can reach $15 \%[3]$.

Early intervention requires an accurate diagnosis and imaging techniques enable both a positive diagnosis, as well as arthrocentesis and liquid analysis, the "gold standard" criteria. Modern imaging methods, such as musculoskeletal ultrasonography, might add important advantages in both diagnosis and management of the rheumatic diseases. Musculoskeletal ultrasound (US) is a fast, reproducible, low-cost technique, which can be easily

This is an open-access article distributed under the terms of a Creative Commons Attribution-NonCommercial-ShareAlike 4.0 International Public License, which permits unrestricted use, adaptation, distribution and reproduction in any medium, non-commercially, provided the new creations are licensed under identical terms as the original work and the original work is properly cited. 
used in any articular pathology for differential diagnosis and joint aspiration, in case of articular effusion [4-8]. Along with laboratory studies, enables an early diagnosis and proper therapeutic approach, as cartilage damage occurs very fast after the onset of infection.

\section{Aim}

We report the case of a 70-year-old patient, with history of RA, DM and persistent left malum perforans in the last year, with development of a severe and debilitating $S$. aureus-related SA of the left ankle, which posed significant therapeutic challenges.

\section{ㅁ Case presentation}

A 70-year-old patient was admitted to the Department of Rheumatology, Emergency County Hospital, Craiova, Romania, in May 2018, following an ongoing and worsening pain and swelling of the left ankle in the last two months. The patient has a long history of type II DM, with secondary neurological and vascular complications. Other past disease history included a coronary angioplasty 14 years ago and arterial hypertension. Home medication consisted of antihypertensive drugs, oral antidiabetics, and platelet antiaggregant. He has had persistent arthralgias in the hands for the past four years which we're recently labeled as seropositive RA.

Since diagnosis of RA, the patient was started on Leflunomide and Sulfasalazine immunosuppressive drugs.

He developed a plantar lesion at the ball of the left foot, in the past one year, which was labeled as malum perforans in the setting of DM. The ulceration has since persisted and started to display features of an infectious process with purulent discharge after four months. At that time, the patient was managed in an Orthopedic Department for local debridement and close follow-up. Cultures drawn from the tissue were positive for S. aureus. After an antibiotic course, the apparent infectious features were remitted, but the long-lasting open wound failed to improve.

Upon admission in the Rheumatology Clinic, the patient presented with intense pain in the left ankle, with marked joint instability, limited range of motion and need of a walking aid and ankle orthosis. On physical examination, the ankle joint showed overall swelling, erythema, tenderness, and increased pain during movement. Local weightbearing could not be performed. Other joint symptoms were not evident, with no apparent signs of an active RA. Results of blood tests on admission showed an important inflammatory syndrome, with high values for both erythrocyte sedimentation rate (ESR) and C-reactive protein (CRP), and a moderate anemia, with normal erythrocyte indices. The results are shown in Table 1 . The patient underwent multiple US examinations for guided fluid aspiration and follow-up. An X-ray and magnetic resonance imaging (MRI) of the left ankle were also performed.

\section{Imaging evaluation}

Musculoskeletal US was the primary imaging technique used to define the location and extent of the infectious process. On admission, US assessment revealed significant changes related to a potential joint infection (Figures 1 and 2). Anterior scans displayed increased joint effusion of the tibiotalar joint, with extension on the lateral aspect of the joint. Marked tenosynovitis of the peroneus longus and peroneus brevis tendons was also detected. The joint effusion displayed an overall inhomogeneous hypoechogenic content with scattered hyperechoic spots. Following close examination of the extent of the joint collection, an US-guided fluid aspiration was performed. A total of $30 \mathrm{~mL}$ of joint fluid was evacuated and microscopic analysis of the sample drawn displayed evident purulent features. The sample was sent for further cytology and microbiology studies. Confirmation of a $S$. aureus etiology through positive joint fluid culture allowed for a targeted antibiotic therapy course. During the follow-up period, the patient underwent additional US exams. Although no significant change was seen in the following scans regarding the quantity of joint effusion, further fluid aspiration failed to obtain significant samples due to a very thickened content. A slight benefit for better aspiration was obtained through joint lavage using saline solution. Left ankle MRI scans confirmed the presence of arthritis and tenosynovitis with additional diffuse bone edema of the tibial epiphysis, talus bone (Figures 3 and 4). X-ray image displayed important structural changes in the form of cortical irregularities, peri-articular demineralization, and joint space-narrowing.

Table 1 - Laboratory investigations

\begin{tabular}{|c|c|c|}
\hline Analysis & Result & Reference range \\
\hline Hemoglobin & $\begin{array}{c}8.55 \mathrm{~g} / \mathrm{dL} \\
\left({ }^{*} \text { normal erythrocyte indices) }\right.\end{array}$ & $12.6-17.4 \mathrm{~g} / \mathrm{dL}$ \\
\hline Leukocyte count & $7.418 / \mathrm{mm}^{3}$ & $4000-10000 / \mathrm{mm}^{3}$ \\
\hline CRP & $152 \mathrm{mg} / \mathrm{L}$ & $0-5 \mathrm{mg} / \mathrm{L}$ \\
\hline ESR & $86 \mathrm{~mm} / 1 \mathrm{~h}$ & $1-10 \mathrm{~mm} / 1 \mathrm{~h}$ \\
\hline Fasting glucose & $122 \mathrm{mg} / \mathrm{dL}$ & $70-110 \mathrm{mg} / \mathrm{dL}$ \\
\hline Urea & $37 \mathrm{mg} / \mathrm{dL}$ & $18-55 \mathrm{mg} / \mathrm{dL}$ \\
\hline Creatinine & $0.84 \mathrm{mg} / \mathrm{dL}$ & $0.72-1.25 \mathrm{mg} / \mathrm{dL}$ \\
\hline AST & $20 \mathrm{U} / \mathrm{L}$ & $5-34 \mathrm{U} / \mathrm{L}$ \\
\hline ALT & $16 \mathrm{U} / \mathrm{L}$ & $3-55 \mathrm{U} / \mathrm{L}$ \\
\hline \multicolumn{3}{|c|}{ Joint fluid analysis } \\
\hline Cytology & \multicolumn{2}{|c|}{$\begin{array}{l}\text { Numerous leukocytes, mononuclear cells, } \\
\text { erythrocytes, and abundant detritus. }\end{array}$} \\
\hline Culture & \multicolumn{2}{|c|}{ Positive for Staphylococcus aureus. } \\
\hline
\end{tabular}

ALT: Alanine aminotransferase; AST: Aspartate aminotransferase; CRP: C-reactive protein: ESR: Erythrocyte sedimentation rate.

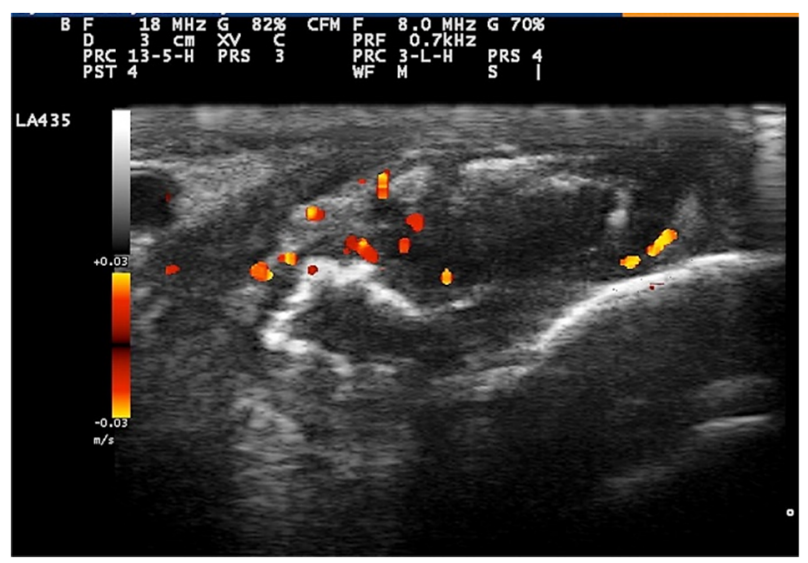

Figure 1 - Peroneus tendon sheath filled with heterogeneous material and marked irregularities of distal fibular bone cortical. Ultrasound image, short axis. 


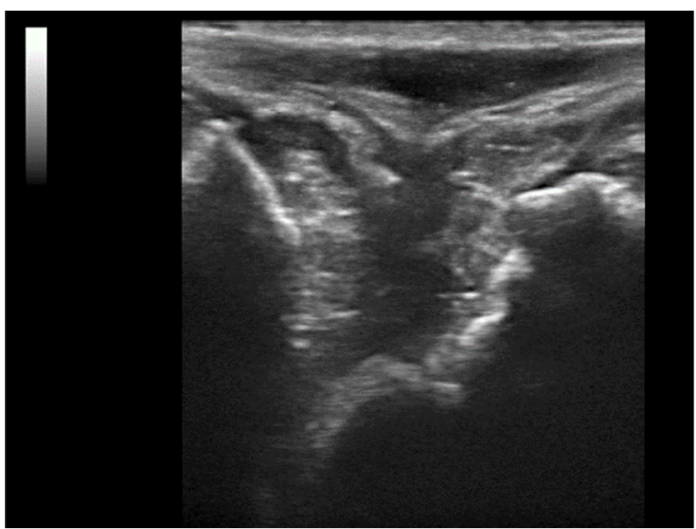

Figure 2 - Tibiotarsal synovitis with overall inhomogeneous joint collection, with hyperechoic floating conglomerates. Ultrasound image, long axis.

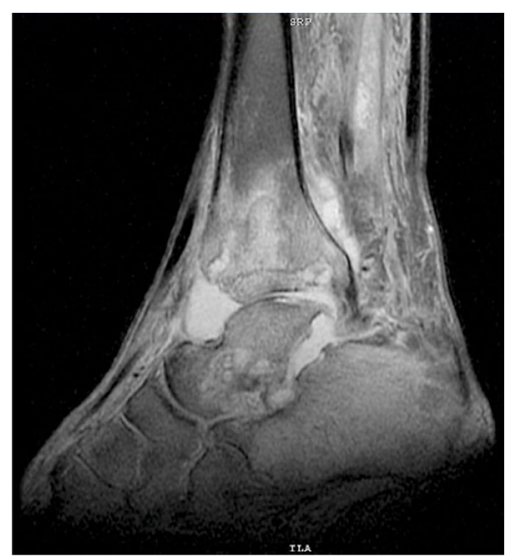

Figure 3 - Hyperintense signal suggesting marked bone marrow edema and intense tibiotarsal and subtalar joint synovitis. MRI of the ankle, sagittal FS PD-FSE. FS PD-FSE: Fat-suppressed proton-density fast-spin-echo; MRI: Magnetic resonance imaging.

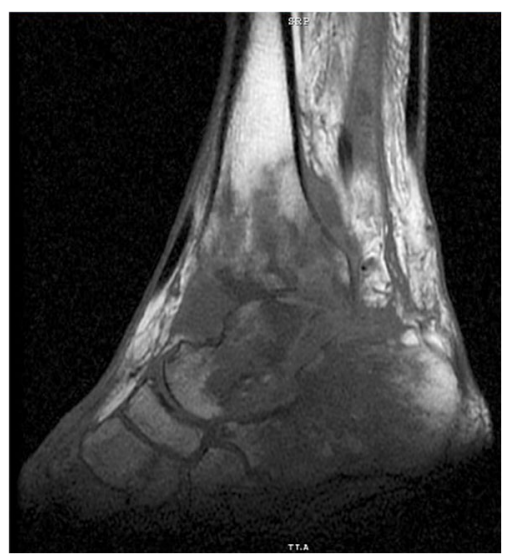

Figure 4 - Hypointense signal at the level of the tibia, talus, and calcaneus bone. Marked lysis of the aforementioned bones. MRI of the ankle, sagittal, T1 (longitudinal relaxation time) FSE image. FSE: Fastspin-echo; MRI: Magnetic resonance imaging.

\section{Management and outcome}

Antibiotic therapy was initiated in accordance with culture sensibility tests. We opted for a 14-day intravenous course of fluoroquinolone - Moxifloxacin $800 \mathrm{mg} /$ day. Nonsteroidal anti-inflammatory, analgesic and Sulfasalazine therapy was maintained, with discontinuation of Leflunomide.
The patient displayed clinical improvement after one week, with pain reduction and better range of joint movement. Unfortunately, the apparent clinical response did not correlate with the follow-up US scans, which revealed persistent joint and peritendinous effusion.

The plantar lesion showed no development of local inflammation or infection, and surgical approach was not recommended at that point.

Before ending the two-week antibiotic course, the patient developed acute decompensated heart failure, massive pleural effusion and was referred to the Cardiology Department. The opportunity for surgery was once again postponed following this flare and fluoroquinolone therapy was extended for additional two weeks. The patient was lost to follow-up after discharge from Cardiology Department. Short- and long-term outcome was unfavorable with both treatment unresponsiveness and comorbidity burden posing considerable difficulties.

\section{ㅁ Discussions}

Patients diagnosed with SA usually present a monoarticular pattern of joint involvement and associate one or more risk factors. SA with absence of risk factors is reported in $22 \%$ of cases [9]. An underlying joint pathology, such as RA, is the most common risk factor associated with SA. In general, up to $47 \%$ of SA patients have a history of joint pathology [10]. RA in particular poses multiple risks for bacterial infection, because of joint lesions, poor skin condition and immunosuppression. Studies on large case series of patients with SA, cite the presence of RA in $10-40 \%$ of SA cases [11]. In the presence of an existing RA or prosthetic joint, incidence of SA rises from 10 to 70 cases per 100000 individuals [12]. Data from a population-based study by Doran et al. [13] indicate to a higher risk for severe infections requiring hospitalization in RA patients versus non-RA patients, with a rate ratio of 1.88 . SA in particular was linked to the highest incidence risk in this study, with a rate ratio of 21.66 [1]. DM can also act as a double risk factor, through both compromised immunity and development of skin ulcers $[9,14,15]$. Our patient was considered a high-risk case, in which combined risk factors related to both RA and DM led to a high susceptibility for joint infection.

The diagnosis of SA in individuals with RA is usually delayed because of flare-like presentation of the infectious disease.

A poor prognosis observed in most of RA patients can be related to a difficult early diagnosis and to the immunocompromised status, due to disease itself or immunosuppressive treatment.

Although RA is a well-established risk factor for SA, the independent effect of specific disease-modifying antirheumatic drugs (DMARDs) is a debatable topic. Edwards et al., in 2016, compared incidence rate ratios of SA for DMARD and non-DMARD RA patients [16]. The study reported significant differences only for Sulfasalazine (1.74) and Prednisolone (2.94), while other DMARDs, including Methotrexate and Leflunomide, did not show significant effects. Upon further analysis, the authors concluded that RA in itself should be considered a more relevant risk factor than any drug in particular and that 
the apparent link to DMARD usage can be underlined by a high disease activity [16]. The most frequent infectious events related to DMARD use are the respiratory tract infections. Wolfe et al. (2006), reported specific RA treatment risks for lung infection and labeled Leflunomide as an independent risk factor for pneumonia [odds ratio (OR) 1.2] [17]. RA patients on combination DMARD therapy have an increased the incidence of fever/infection as opposed to individuals on monotherapy [18]. There is a general concern about the DMARD maintenance throughout an infectious episode. It is a common practice to discontinue DMARDs in the presence of a serious infectious event which requires hospitalization and intravenous antibiotics, with resumption of therapy after recovery [19].

Imaging studies are an integral part of the management of SA patients. MRI has a very high sensitivity, of nearly $100 \%$, in diagnosing joint effusion, synovitis and bone or cartilage destruction [20]. Musculoskeletal US provides also a significant diagnostic performance with further advantages regarding availability, low cost and crucial aid in guided fluid aspiration or synovial biopsy [21]. Presence of joint fluid is the hallmark feature of SA on US. Depending on the time from onset, joint effusion may be hypoechoic or hyperechoic with blurred demarcation from synovial tissue [22]. Gaigneux et al. reported high prevalence of over $90 \%$ for both joint effusion and synovitis on US in a group of 34 patients with SA [20]. These changes persisted in a significant proportion of cases even after three months of therapy. Synovial hypervascularity was also common $(64.3 \%)$ and could be associated with functional outcome. Residual lesions including synovial thickening, cellulitis and bone edema can be detected even after infection eradication [23].

The cornerstone of SA treatment is the adequate drainage through arthrocentesis or surgical approach coupled with parenteral antibiotics. If initial needle aspiration does not provide fast relief or the purulent fluid is too thick for aspiration, open drainage through arthroscopy or arthrotomy are strongly recommended. Some studies found superior results for surgical approach in deeper joint, such as the hip or shoulder [24-26]. Patients who are unfit for surgery can benefit also from joint washing by means of an irrigationdrainage system, especially in the knee joint [27]. In our approach, joint drainage was improved through saline solution irrigation and aspiration. Intra-articular corticosteroids are generally avoided in the setting of SA. Some authors address the benefit of this approach in patients which followed adequate systemic antibiotic and have persistent synovitis with sterile joint fluid and blood culture $[28,29]$.

A complex imagistic and laboratory assessment allowed us to establish an accurate diagnosis, but the several associated risk factors and consecutive complications did not allow us to apply all the necessary therapeutic measures, making the case difficult to have a good short- and longterm good prognosis [30, 31].

\section{ㅁ Conclusions}

Early diagnosis and prompt antibiotic treatment are crucial for SA management. Outcome is generally worse in the presence of an underlying joint pathology. The association and interrelation between different comorbidities (such as hypertension, diabetes, or obesity), chronic systemic inflammation (e.g., CRP level, disease activity), and RA medication, is sometimes difficult to understand and to address in daily practice and this case report highlights multiple toils encountered in a SA patient with RA on immunosuppressive therapy and complicated DM. Several factors contributed to the high risk of infectious disease, limitation of treatment and insufficient response.

\section{Conflict of interests}

The authors declare that they have no conflict of interests.

\section{Authors' contribution}

Andreea Lili Bărbulescu and Ştefan Cristian Dinescu have equally contributed to this paper.

\section{References}

[1] Carpenter CR, Schuur JD, Everett WW, Pines JM. Evidencebased diagnostics: adult septic arthritis. Acad Emerg Med, 2011, 18(8):781-796. https://doi.org/10.1111/j.1553-2712.2011. 01121.x. Erratum in: Acad Emerg Med, 2011, 18(9):1011. PMID: 21843213 PMCID: PMC3229263

[2] Shirtliff ME, Mader JT. Acute septic arthritis. Clin Microbiol Rev, 2002, 15(4):527-544. https://doi.org/10.1128/CMR.15. 4.527-544.2002 PMID: 12364368 PMCID: PMC126863

[3] Gupta MN, Sturrock RD, Field M. A prospective 2-year study of 75 patients with adult-onset septic arthritis. Rheumatology (Oxford), 2001, 40(1):24-30. https://doi.org/10.1093/rheuma tology/40.1.24 PMID: 11157138

[4] Filippou G, Scirè CA, Adinolfi A, Damjanov NS, Carrara G, Bruyn GAW, Cazenave T, D'Agostino MA, Delle Sedie A, Di Sabatino V, Diaz Cortes ME, Filippucci E, Gandjbakhch F, Gutierrez M, Maccarter DK, Micu M, Möller Parera I, Mouterde G, Mortada MA, Naredo E, Pineda C, Porta F, Reginato AM, Satulu I, Schmidt WA, Serban T, Terslev L, Vlad V, Vreju FA, Zufferey $P$, Bozios $P$, Toscano $C$, Picerno V, lagnocco A. Identification of calcium pyrophosphate deposition disease (CPPD) by ultrasound: reliability of the OMERACT definitions in an extended set of joints - an international multiobserver study by the OMERACT Calcium Pyrophosphate Deposition Disease Ultrasound Subtask Force. Ann Rheum Dis, 2018, 77(8):1194-1199. https://doi.org/10.1136/annrheumdis-2017212542 PMID: 29535120

[5] Barbulescu AL, Ciurea PL, Mitran C, Chisalau BA, Parvanescu CD, Firulescu SC, Balasoiu M, Boldeanu MV, Popoviciu H, Vreju FA. High frequency ultrasonography of the hand versus anti-RA33 evaluation in early rheumatoid arthritis - a pilot study. Med Ultrason, 2017, 19(2):166-171. https://doi.org/10.11152/mu-995 PMID: 28440350

[6] AIOayan LI, Zawawi AH. Musculoskeletal manifestations among diabetic patients in Saudi Arabia. J Family Med Prim Care, 2020, 9(11):5597-5600. https://doi.org/10.4103/jfmpc.jfmpc_ 654_20 PMID: 33532401 PMCID: PMC7842468

[7] Kiely PDW, Lloyd ME. Ankle arthritis - an important signpost in rheumatologic practice. Rheumatology (Oxford), 2021, 60(1):23-33. https://doi.org/10.1093/rheumatology/keaa531 PMID: 33097958 PMCID: PMC7785314

[8] Firulescu SC, Tudoraşcu DR, Pârvănescu CD, Chisălău AB, Bastian AE, Efrem IC, Bărbulescu AL, Forţofoiu MC, Criveanu C, Ionescu P, Dinescu ŞC, Tudorancea AD, Ciurea PL, Vreju AF. The role of skin and muscle biopsy in the diagnosis of main connective tissue diseases. Rom J Morphol Embryol, 2018, 59(1):55-64. PMID: 29940612

[9] Kaandorp CJ, Van Schaardenburg D, Krijnen P, Habbema JD, van de Laar MA. Risk factors for septic arthritis in patients with joint disease. A prospective study. Arthritis Rheum, 1995, 38(12):1819-1825. https://doi.org/10.1002/art.1780381215 PMID: 8849354

[10] Ross JJ. Septic arthritis of native joints. Infect Dis Clin North Am, 2017, 31(2):203-218. https://doi.org/10.1016/j.idc.2017. 01.001 PMID: 28366221

[11] Goldenberg DL. Infectious arthritis complicating rheumatoid arthritis and other chronic rheumatic disorders. Arthritis Rheum, 
1989, 32(4):496-502. https://doi.org/10.1002/anr.1780320422 PMID: 2650687

[12] Weston VC, Jones AC, Bradbury N, Fawthrop F, Doherty M Clinical features and outcome of septic arthritis in a single UK Health District 1982-1991. Ann Rheum Dis, 1999, 58(4):214219. https://doi.org/10.1136/ard.58.4.214 PMID: 10364899 PMCID: PMC1752863

[13] Doran MF, Crowson CS, Pond GR, O'Fallon WM, Gabriel SE. Frequency of infection in patients with rheumatoid arthritis compared with controls: a population-based study. Arthritis Rheum, 2002, 46(9):2287-2293. https://doi.org/10.1002/art. 10524 PMID: 12355475

[14] Pucino V, Certo M, Varricchi G, Marone G, Ursini F, Rossi FW De Paulis A, Mauro C, Raza K, Buckley CD. Metabolic checkpoints in rheumatoid arthritis. Front Physiol, 2020, 11:347. https://doi.org/10.3389/fphys.2020.00347 PMID: 32362840 PMCID: PMC7180190

[15] Gobao VC, Alfishawy M, Smith C, Byers KE, Yassin M, Urish KL, Shah NB. Risk factors, screening, and treatment challenges in Staphylococcus aureus native septic arthritis. Open Forum Infect Dis, 2020, 8(1):ofaa593. https://doi.org/ 10.1093/ofid/ofaa593 PMID: 33511230 PMCID: PMC7813160

[16] Edwards CJ, Cooper C, Fisher D, Field M, van Staa TP, Arden NK. The importance of the disease process and diseasemodifying antirheumatic drug treatment in the development of septic arthritis in patients with rheumatoid arthritis. Arthritis Rheum, 2007, 57(7):1151-1157. https://doi.org/10.1002/art. 23003 PMID: 17907232

[17] Wolfe F, Caplan L, Michaud K. Treatment for rheumatoid arthritis and the risk of hospitalization for pneumonia: associations with prednisone, disease-modifying antirheumatic drugs, and anti-tumor necrosis factor therapy. Arthritis Rheum, 2006, 54(2):628-634. https://doi.org/10.1002/art.21568 PMID: 16447241

[18] Aletaha D, Kapral T, Smolen JS. Toxicity profiles of traditional disease modifying antirheumatic drugs for rheumatoid arthritis. Ann Rheum Dis, 2003, 62(5):482-486. https://doi.org/10.1136/ ard.62.5.482 PMID: 12695166 PMCID: PMC1754550

[19] Taylor PC, Atzeni F, Balsa A, Gossec L, Müller-Ladner U, Pope J. The key comorbidities in patients with rheumatoid arthritis: a narrative review. J Clin Med, 2021, 10(3):509. https:// doi.org/10.3390/jcm10030509 PMID: 33535498 PMCID: PMC7867048

[20] Gaigneux E, Cormier G, Varin S, Mérot O, Maugars Y, Le Goff B. Ultrasound abnormalities in septic arthritis are associated with functional outcomes. Joint Bone Spine, 2017, 84(5):599_ 604. https://doi.org/10.1016/j.jbspin.2017.02.002 PMID: 28219656

[21] Thom C, Ahmed A, Kongkatong M, Moak J. Point-of-care hip ultrasound leads to expedited results in emergency department patients with suspected septic arthritis. J Am Coll Emerg Physicians Open, 2020, 1(4):512-520. https://doi.org/10.1002/ emp2.12167 PMID: 33000078 PMCID: PMC7493574

[22] Chau CLF, Griffith JF. Musculoskeletal infections: ultrasound appearances. Clin Radiol, 2005, 60(2):149-159. https://doi.org/ 10.1016/j.crad.2004.02.005 PMID: 15664569
[23] Bierry G, Huang AJ, Chang CY, Torriani M, Bredella MA. MRI findings of treated bacterial septic arthritis. Skeletal Radiol, 2012, 41(12):1509-1516. https://doi.org/10.1007/s0 0256-012-1397-2 PMID: 22430565

[24] Böhler C, Pock A, Waldstein W, Staats K, Puchner SE, Holinka J, Windhager R. Surgical treatment of shoulder infections: a comparison between arthroscopy and arthrotomy. J Shoulder Elbow Surg, 2017, 26(11):1915-1921. https://doi. org/10.1016/j.jse.2017.04.001 PMID: 28601485

[25] Flores-Robles BJ, Jiménez Palop M, Sanabria Sanchinel AA, Andrus RF, Royuela Vicente A, Sanz Pérez MI, Espinosa Malpartida M, Ramos Giráldez C, Merino Argumanez C, Villa Alcázar LF, Andréu Sánchez JL, Godoy Tundidor H, Campos Esteban J, Sanz Sanz J, Barbadillo Mateos C, Isasi Zaragoza C, Mulero Mendoza J. Medical versus surgical approach to initial treatment in septic arthritis: a single Spanish Center's 8-year experience. J Clin Rheumatol, 2019, 25(1): 4-8. https://doi.org/10.1097/RHU.0000000000000615 PMID: 29215382 PMCID: PMC7654724

[26] Turner EHG, Lang MDH, Spiker AM. A narrative review of the last decade's literature on the diagnostic accuracy of septic arthritis of the native joint. J Exp Orthop, 2021, 8(1):3. https:// doi.org/10.1186/s40634-020-00315-w PMID: 33423115 PMCID: PMC7797010

[27] Khoo SS, Loi KW, Tan KT, Suhaeb AR, Simmrat S. Bedside continuous irrigation and drainage as an interim local treatment for septic arthritis of the knee in the medically unstable patient: a case report. Malays Orthop J, 2015, 9(2):57-59. https:// doi.org/10.5704/MOJ.1507.003 PMID: 28435613 PMCID: PMC5333672

[28] Lane SE, Merry P. Intra-articular corticosteroids in septic arthritis: beneficial or barmy? Ann Rheum Dis, 2000, 59(3):240. https:// doi.org/10.1136/ard.59.3.240 PMID: 10777422 PMCID: PMC 1753092

[29] Kwon HY, Cha B, Im JH, Baek JH, Lee JS. Medical management of septic arthritis of sternoclavicular joint: a case report. Medicine (Baltimore), 2020, 99(44):e22938. https://doi.org/ 10.1097/MD.0000000000022938 PMID: 33126360 PMCID: PMC7598876

[30] Dinescu SC, Dumitrascu RM, Firulescu SC, Parvanescu CD, Chisalau AB, Criveanu C, Barbulescu AL, Musetescu AE, Rosu A, Ciurea P, Vreju AF. Acute nongonococcal septic arthritis of the ankle in high risk patient with diabetic foot and rheumatoid arthritis. World Congress on Osteoporosis, Osteoarthritis and Musculoskeletal Diseases (WCO-IOFESCEO 2019), Paris, 4-7 April 2019: Posters Abstracts. Osteoporos Int, 2019, 30(Suppl 2):S745. https://doi.org/10. 1007/s00198-019-04993-w

[31] Rasmussen L, Bell J, Kumar A, Heckman MG, Lesser E, Whalen J, Shi GG, Ledford C, Wilke B. A retrospective review of native septic arthritis in patients: can we diagnose based on laboratory values? Cureus, 2020, 12(6):e8577. https://doi. org/10.7759/cureus.8577 PMID: 32670713 PMCID: PMC 7358919

\section{Corresponding authors}

Mihail Virgil Boldeanu, Lecturer, MD, PhD, Department of Immunology, University of Medicine and Pharmacy of Craiova, 661 May Avenue, 200628 Craiova, Dolj County, Romania; Phone +40724-515 810, e-mails: laborator.imunologie@umfcv.ro, boldeanumihailvirgil@yahoo.com

Adina Turcu-Ştiolică, Associate Professor, MD, PhD, Department of Biostatistics, University of Medicine and Pharmacy of Craiova, 2 Petru Rareş Street, 200349 Craiova, Dolj County, Romania; Phone +40726-270 295, e-mail: adina.turcu@gmail.com 